\title{
Embryonic stem cells as sources of donor-independent platelets
}

\author{
Matthew C. Canver, ${ }^{1}$ Daniel E. Bauer, ${ }^{1,2,3}$ and Stuart H. Orkin ${ }^{1,2,3,4}$ \\ ${ }^{1}$ Harvard Medical School, Boston, Massachusetts, USA. ²Division of Hematology/Oncology, Boston Children's Hospital, Boston, Massachusetts, USA. ${ }^{3}$ Department of Pediatric Oncology, Dana-Farber Cancer \\ Institute, Boston, Massachusetts, USA. ${ }^{4}$ Howard Hughes Medical Institute, Boston, Massachusetts, USA.
}

\begin{abstract}
The creation of a donor-independent source of platelets has been challenging; however, recent advances show growing promise for alternative platelet sources. Pluripotent stem cells have the capacity to differentiate into mature megakaryocytes with the ability to produce functional platelets. In this issue of $J C l$, Noh et al. provide a proof-of-principle demonstration that embryonic stem cells can be used to produce platelets on a clinical scale by controlling the level of the transcription factor GATA1. This study emphasizes the importance of precise regulation of gene expression for regenerative medicine applications.
\end{abstract}

\section{Thrombocytopenia and the need for alternative platelet sources}

The blood system comprises a hierarchy of rare, largely quiescent, self-renewing hematopoietic stem cells (HSCs) and highly proliferative lineage-committed progenitors and precursors in the bone marrow, which yield a continual supply of mature blood cells of diverse lineages (1). The nearly one trillion platelets present in circulation derive from bone marrow-resident, multinucleated megakaryocytes, which in turn derive from bipotential megakaryocyte-erythroid progenitors (MEPs). The master transcription factor GATA1 is required for maturation of MEPs into both megakaryocytic and erythroid lineages (1, 2). It is estimated that each megakaryocyte produces approximately $10^{3}$ to $10^{4}$ platelets, which have half-lives of only about three days in the circulation (3-6). Reduction in the number of circulating platelets, termed thrombocytopenia, is a common clinical condition with a variety of etiologies, including infectious and heritable disease, malignancy, liver failure, chemotherapy, and medication side effects (1, 4, 7-9).
Transfusion of donor-derived platelets is employed with increasing frequency to treat patients with thrombocytopenia and places a high demand on platelet donation (10). Over two million platelet units are transfused annually in the United States $(8,10)$; however, there are multiple factors that hinder platelet transfusion. Donor platelets have only a three- to seven-day shelf life, limiting the ability to store and transport these cells and generating substantial waste through the disposal of aged platelets. Intermittent shortages arise due to a mismatch between supply and demand (11, 12). Further, donor-derived platelets carry the inherent risk of viral transmission and bacterial contamination due to extended room temperature storage (8). Together, these limitations have motivated the development of sustainable donor-independent platelet sources to meet the growing need for these cells $(7,11)$.

Pluripotent embryonic stem (ES) cells or induced pluripotent stem (iPS) cells have been proposed as a potential donor-independent source of platelets. One vision is that either ES cells or iPS cells could be used to produce a renew-

\section{Related Article: p. 2369}

able and immortalized progenitor-like cell line that retains the capacity to differentiate into mature megakaryocytes, which in turn would produce functional platelets. To date, attempts to employ this strategy have suffered from a poor output of PS cell-derived megakaryocytes. Moreover, inefficient generation of platelets from megakaryocytes in vitro has been an additional challenge $(4,6,11,13,14)$.

\section{The GATA switch and} regulation of megakaryopoiesis The study of the GATA transcription factors GATA1 and GATA2 has provided insight into normal and disease-associated megakaryopoiesis. While GATA2 is highly expressed in early stem and progenitor cells, megakaryopoiesis and erythropoiesis are accompanied by a concurrent downregulation of GATA2 and upregulation of the closely related GATA1, a process referred to as GATA switching. The switch to GATA1 reflects a dependence on this key factor for terminal differentiation of megakaryocytes and erythroid cells $(15,16)$. Loss-of-function mutations in the gene encoding GATA1 underlie several human conditions, including congenital thrombocytopenias and anemias and the aberrant expansion of MEPs that is characteristic of Down syndrome-associated transient myeloproliferative disorder (17-19). Previous work demonstrated that loss of GATA1 in mouse ES cells (mES cells) leads to production of selfrenewing MEP-like cells under appropriate ex vivo differentiation conditions (2). Maturation recurs in these cells upon introduction of GATA1; however, this is not enough to complete differentiation into functional megakaryocytes, as evidenced by a failure to produce platelets in vivo.

In this issue, Noh et al. report on their hypothesis that the failure to achieve full differentiation reflects inappropriate regulation of GATA1 (20). Noh and colleagues developed an elegant system in which 


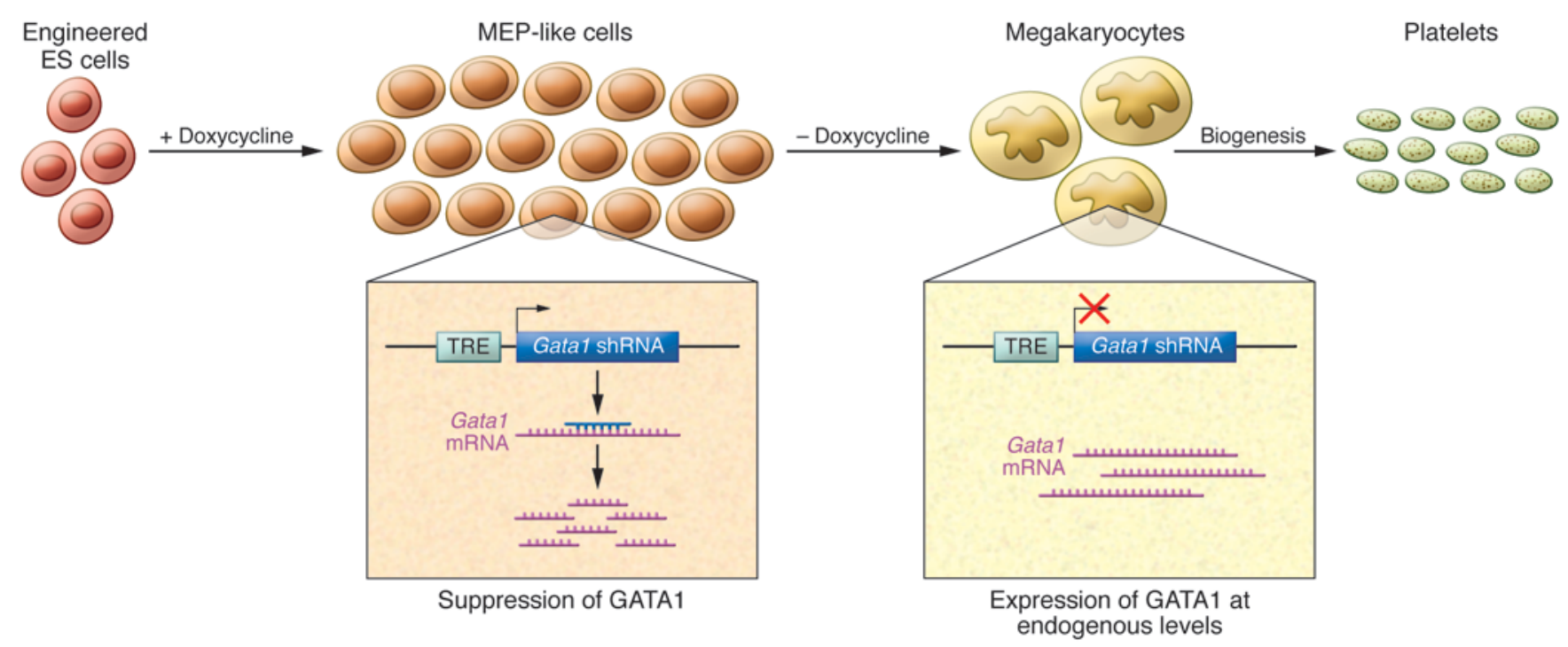

Figure 1. Strategy for clinical scale production of platelets ex vivo. Engineered ES cells harbor a doxycycline-inducible promoter that mediates the expression of an shRNA that targets Gata1 mRNA, resulting in degradation of the full-length transcript. Addition of doxycycline induces shRNA-mediated GATA1 suppression, leading to logarithmic proliferation of MEP-like cells (G1ME2 cells). The restoration of GATA1 expression upon doxycycline withdrawal supports differentiation of MEP-like cells into mature megakaryocytes capable of producing functional platelets. TRE, tetracylcine response element.

GATA1 is transiently repressed in mES cells with an shRNA that is under the control of a doxycycline-responsive promoter, allowing release of GATA1 repression by removal of doxycycline. Moreover, with this scheme, GATA1 regulation proceeds via endogenous control mechanisms once it is no longer being repressed. Indeed, compared with previous attempts, which employed Gata1 knockout and retroviralmediated reexpression (2), this inducible repression system provides a gradual induction of Gata 1 mRNA that closely recapitulates the differentiation of fetal liver progenitors into megakaryocytes in vitro with a commensurate downregulation of GATA2 as GATA1 is induced along with expected alterations of downstream target gene expression. In the presence of doxycycline, which induces shRNA-mediated GATA1 suppression, these ex vivo-differentiated mES cells exhibited logarithmic growth for over 40 days as MEP-like cells (referred to as G1ME2 cells), resulting in an approximately $10^{13}$-fold expansion in cell number. Upon doxycycline withdrawal, which restores GATA1, approximately $20 \%$ of G1ME2 cells differentiated into cells with morphological and expression patterns that were consistent with mature megakaryocytes (20). Furthermore, these G1ME2-derived megakaryocytes generated proplatelet processes, a hallmark of platelet generation (Figure 1).

\section{A fine-tuning of gene expression provides the key}

Following successful production of mature megakaryocytes ex vivo, Noh et al. turned to mouse models to validate the quality of their G1ME2-derived megakaryocytes. Recent studies have demonstrated that infusion of mature megakaryocytes into mice leads to the production of functional platelets (21). Infusion of G1ME2-derived megakaryocytes into mice led to observable platelet production. Furthermore, an arteriole laser injury model revealed that these platelets are incorporated into functional thrombi at a rate that is indistinguishable from the rates of incorporation of either platelets produced from fetal liver-derived megakaryocytes or platelets isolated from donor mice. These experiments provide in vivo evidence that G1ME2-derived megakaryocytes can produce platelets able to home to sites of injury and participate in thrombus formation (20).

These results from the study by Noh and colleagues have several important implications. First, they emphasize that proper cellular differentiation relies not only on the absence or presence of the correct transcriptional regulatory factors, but also requires appropriate levels and timing of expression. These results also suggest that protocols for cellular engineering and reprogramming may benefit from relying on and incorporating endogenous gene regulatory mechanisms to any extent pos- sible. In addition, gene therapy approaches often utilize strong promoters, such as those driving expression of viral genes or mammalian housekeeping genes, to maximize gene expression. However, the findings of Noh et al. emphasize that too much expression may be just as problematic as too little expression to restore normal function. The need to fine-tune gene expression is likely to extend to ORF overexpression and clustered regularly interspaced palindromic repeat (CRISPR) gene activation experiments, which may fail to identify key regulators due to inappropriate spatiotemporal and/or nonphysiologic levels of gene expression.

The study by Noh and colleagues brings the field a step closer to human donor-independent sources of platelets. In order to achieve clinical utility, platelets must be produced in large quantities $(4,11)$, as currently one unit for transfusion contains 3 to $4 \times 10^{11}$ platelets (8). With the assumption that each megakaryocyte can produce $10^{3}$ platelets $(3,4,6), 3$ to $4 \times 10^{8}$ megakaryocytes would be required for a single platelet transfusion and more than 6 to $8 \times 10^{14}$ megakaryocytes would be needed to replace the necessity of donor-derived platelets across the United States. Noh et al. demonstrated that each G1ME2-derived megakaryocyte produces approximately 40 platelets with a half-life of five to six hours when transferred into mouse models (20); however, the incred- 
ible expansion of G1ME2 cells allows for the production of clinical levels of platelets, despite reduced platelet output per megakaryocyte. This reduced ability of the G1ME2-derived megakaryocytes to produce platelets and shortened half-life of these platelets may reflect an incomplete differentiation of G1ME2 cells or an intrinsic biological limitation as the result of deriving G1ME2 cells from cells of embryonic origin and not adult cells. The possibility of a developmental stage restriction represents a potential roadblock to using PS cells derived from embryonic sources for the production of many adult cell types. A clearer understanding of the molecular mechanisms of ontogeny may inform attempts to more successfully engineer robust in vitro models of adult megakaryopoiesis and platelet production.

\section{Conclusions and future directions}

The next step toward clinical translation of the work by Noh and colleagues is an extension of this strategy to human ES cells (hES cells) and iPS cells. Cross-species differences between mES cells and hES cells are appreciable (22); however, the similar transcriptional networks involved in murine and human hematopoiesis suggest that inducible suppression and restoration of GATA1 may be applicable in human cells. The low platelet outputs from hES cell-derived megakaryocytes are an additional hurdle that must be overcome before clinical realization $(23,24)$. Moreover, the use of hES cells as a megakaryocyte source also warrants discussion of both safety and ethics. Fortunately, as platelets lack nuclear DNA, platelet products can be irradiated prior to transfusion, which enhances safety by diminishing the risk of tumorigenesis from residual hES cells (11). Turning to human iPS cells could obviate ethical concerns related to embryonic material. A recent study showed that cooverexpression of BMI1, C-MYC, and BCL$\mathrm{XL}$ led to the immortalization of hES cellor human iPS cell-derived megakaryocyte cell lines, which retained the capacity to produce functional platelets upon shut-off of transgene expression (24). However, other reports have demonstrated concern that infusion of ex vivo-derived megakaryocytes into mouse models results in production of higher quality platelets than can be generated ex vivo, which suggests that current culture conditions may lack essential components necessary for the release of fully functional platelets (25).

Even if technically successful, the cost of clinical scale-up of hES cell/iPS cell-based cellular differentiation protocols represents a substantial challenge to supplanting the existing systems based on altruistic human donors. In essence, the goal would be to make a clinical product that is at least equivalent in quality and cost to donor-derived platelets. In addition, there are several applications of bioengineered platelets in which the final product might actually be superior to existing available products. For example, platelets are efficient vectors for delivery of cargo to sites of vascular injury. It is plausible that by enhancing prothrombotic or antithrombotic properties, engineered platelets could provide novel treatment options for hemorrhagic or thrombotic diseases (26, 27). Thus, with further enhancements to platelet quality and yield, the approach proposed by Noh et al. could be developed into a practical platform in which to produce platelets at clinical scale. We applaud this advance in the challenging path to a sustainable donor-independent platelet supply.

\section{Acknowledgments}

Address correspondence to: Stuart $\mathrm{H}$. Orkin, Dana Farber Cancer Institute, 44 Binney Street, Boston, Massachusetts 02115, USA. Phone: 617.632.3564; E-mail: Stuart_Orkin@dfci.harvard.edu.

1. Orkin SH, Zon LI. Hematopoiesis: an evolving paradigm for stem cell biology. Cell. 2008;132(4):631-644.

2. Stachura DL, Chou ST, Weiss MJ. Early block to erythromegakaryocytic development conferred by loss of transcription factor GATA-1. Blood. 2006;107(1):87-97.

3. Kaufman R, Airo R, Pollack S, Crosby W. Circulating megakaryocytes and platelet release in the lung. Blood.1965;26(6):720-731.

4. Avanzi MP, Mitchell WB. Ex vivo production of platelets from stem cells. Br J Haematol. 2014;165(2):237-247.

5. Ault K, Knowles C. In vivo biotinylation demonstrates that reticulated platelets are the youngest platelets in circulation. Exp Hematol. 1995;23(9):996-1001.

6. Eto J. Platelet biogenesis wears silkworm cocoons. Blood. 2015;125(14):2181-2183.

7. Smith BW, Murphy GJ. Stem cells, megakaryocytes, and platelets. Curr Opin Hematol. 2014;21(5):430-437.

8. Kaufman RM, et al.Platelet transfusion: a clinical practice guideline from the AABB. Ann Intern Med.
2015;162(3):205-213.

9. Deutsch VR, Tomer A. Advances in megakaryocytopoiesis and thrombopoiesis: from bench to bedside. Br J Haematol. 2013;161(6):778-793.

10. Whitaker BI, Hinkins S. The 2011 National Blood Collection Utilization Survey Report. United States Department of Health Human Services 2011. Washington DC, USA: The United States Department of Health and Human Services; 2011.

11. Lambert MP, Sullivan SK, Fuentes R, French DL, Poncz M. Challenges and promises for the development of donor-independent platelet transfusions. Blood. 2013;121(17):3319-3324.

12. Williamson LM, Devine DV. Challenges in the management of the blood supply. Lancet. 2013;381(9880):1866-1875.

13. Di Buduo CA, et al. Programmable 3D silk bone marrow niche for platelet generation ex vivo and modeling of megakaryopoiesis pathologies. Blood. 2015;3(6):617-627.

14. Thon JN, et al. Platelet bioreactor-on-a-chip. Blood. 2014;124(12):1857-1868.

15. Bresnick EH, Lee HY, Fujiwara T, Johnson KD, Keles S. GATA switches as developmental drivers. J Biol Chem. 2010;285(41):31087-31093.

16. Doré LC, Chlon TM, Brown CD, White KP, Crispino JD. Chromatin occupancy analysis reveals genome-wide GATA factor switching during hematopoiesis. Blood. 2012;119(16):3724-3733.

17. Nichols KE, et al. Familial dyserythropoietic anaemia and thrombocytopenia due to an inherited mutation in GATA1. Nat Genet. 2000;24(3):266-270.

18. Sankaran VG, et al. Exome sequencing identifies GATA1 mutations resulting in Diamond-Blackfan anemia. JClin Invest. 2012;122(7):2439-2443.

19. Li Z, Godinho FJ, Klusmann JH, Garriga-Canut M, Yu C, Orkin SH. Developmental stageselective effect of somatically mutated leukemogenic transcription factor GATA1. Nat Genet. 2005;37(6):613-619.

20. Noh J-Y, et al. Inducible Gata1 suppression expands megakaryocyte-erythroid progenitors from embryonic stem cells. JClin Invest. 2015;125(6):2369-2374.

21. Fuentes R, et al. Infusion of mature megakaryocytes into mice yields functional platelets. J Clin Invest. 2010;120(11):3917-3922.

22. Ginis I, et al. Differences between human and mouse embryonic stem cells. Dev Biol. 2004;269(2):360-380.

23. Takayama N, Eto K. Pluripotent stem cells reveal the developmental biology of human megakaryocytes and provide a source of platelets for clinical application. Cell Mol Life Sci. 2012;69(20):3419-3428.

24. Nakamura S, et al. Expandable megakaryocyte cell lines enable clinically applicable generation of platelets from human induced pluripotent stem cells. Cell Stem Cell. 2014;14(4):535-548.

25. Wang Y, et al. Comparative analysis of human ex vivo-generated platelets vs. megakaryocytegenerated platelets in mice: A cautionary tale [published online ahead of print April 7, 2015]. Blood. doi:10.1182/blood-2014-08-593053.

26. High K. The leak stops here: platelets as delivery vehicles for coagulation factors. JClin Invest. 2006;116(7):1840-1842.

27. Greineder CF, Howard MD, Carnemolla R, Cines DB, Muzykantov VR. Advanced drug delivery systems for antithrombotic agents. Blood. 2013;122(9):1565-1575. 